hep-th/9810129

LBNL-42248, SLAC-PUB-7970, UCB-PTH-98/46

\title{
On Vanishing Two Loop Cosmological Constants in Nonsupersymmetric Strings
}

\author{
Shamit Kachru ${ }^{1}$ and Eva Silverstein ${ }^{2}$ \\ ${ }^{1}$ Department of Physics \\ University of California at Berkeley \\ Berkeley, CA 94720 \\ and \\ Ernest Orlando Lawrence Berkeley National Laboratory \\ Mail Stop 50A-5101, Berkeley, CA 94720 \\ 2 Stanford Linear Accelerator Center \\ Stanford University \\ Stanford, CA 94309
}

It has recently been suggested that in certain special nonsupersymmetric type II string compactifications, at least the first two perturbative contributions to the cosmological constant $\Lambda$ vanish. Support for perturbative vanishing beyond 1-loop (as well as evidence for the absence of some nonperturbative contributions) has come from duality arguments. There was also a direct 2-loop computation which was incomplete; in this note we explain the deficiency of the previous 2-loop calculation and discuss the complete 2-loop computation in two different models. The corrected analysis yields a vanishing 2-loop contribution to $\Lambda$ in these models.

October 1998 


\section{Introduction}

A new class of non-supersymmetric type II models was recently introduced [1],2]. These models have a simple mechanism for cancellation of the 1-loop vacuum energy (due to a guaranteed bose-fermi degeneracy). A similar class of models was constructed in the freefermionic prescription in [3]. In [1] a heuristic argument suggesting that the cancellation should persist to all higher loops was presented. A broader motivation arising from the correspondence between quantum-mechanical fixed lines and flat dilaton potentials [4] in the AdS/CFT correspondence [5] also suggests that cancellation should occur. Various string duality computations now provide indirect evidence for the absence of certain perturbative (and also certain nonperturbative) contributions to $\Lambda$ in these models [6,2, 3].

In [1] a direct analysis of the 2-loop contribution to the cosmological constant was also presented. It was argued that, in a particular gauge, it cancels pointwise on the moduli space. This was based on an analysis of only the spin-structure-dependent part of the amplitude. This analysis was incomplete. In the gauge we chose, picture changing operators coincide, and their operator product expansion produces poles. Some of these pole pieces cancel between matter and ghost contributions, or constitute total derivatives on the moduli space. In general, however, there can be finite pieces which arise from the Taylor expansion of the spin-structure dependent piece.1

In this note we rectify this situation for both the original model of [1] and for an even simpler model which also enjoys similar properties, and was introduced in [2]. This analysis will also appear in a revised version of [1]. In the simpler model, if we choose the gauge with coincident picture-changing operators, summing over all spin structures produces a high enough order zero in the spin-structure-dependent piece to cancel against surviving poles in the spin-structure-independent factor. We discuss this cancellation in $\S 4$. In $\S 5$, we analyze the Taylor expansion of the original set of models [1]. We show that after summing over spin structures all potentially finite terms either cancel between different terms in the correlator of picture changing operators or vanish directly.

Perturbative string calculations at higher genus are notoriously subtle. In all of our analysis we assume that the prescription [7] (following e.g. [8] and [9]) for the supersymmetric 2-loop GSO phases is correct (in particular, in [10] it was argued that this

1 We thank Greg Moore for pointing out this error and for providing helpful constructive comments. 
prescription leads to the correct phases upon factorization). Adjusting the supersymmetric expression to incorporate the phases and twists of our nonsupersymmetric models is relatively straightforward, and we see that the cancellation persists if we make a further specialization of the gauge choice. Different gauge choices differ by total derivatives on the moduli space as explained in [11,12]. This argument applies twist structure by twist structure in the orbifold theory, as what it uses is deformation of a contour integral of the BRST charge, which is invariant under the orbifold group. The corresponding boundary contributions cancel as discussed in [1]. As explained in [1], issues of modular invariance of the gauge choice are simpler in our models due to shifts involved in the orbifold group elements, which greatly reduce the modular group in a given twist structure.

\section{The Simpler Model}

In addition to the original model of [1], we will also be considering a new model introduced in [2]. Since the description of the new model there is somewhat terse, we introduce it in greater detail here. Like the model of [1], it is an orbifold generated by two elements $F$ and $G$. We start from the type II string compactified on a $T^{6}$ which is a product of six circles at the self-dual radius $R=l_{s} / \sqrt{2}$, where $l_{s}=\sqrt{\alpha^{\prime}}$ is the string scale. $F$ and $G$ act on the left and right moving degrees of freedom of the superstring as

$\begin{array}{lll}S^{1} & F & G \\ 1 & 1 & (s,-1) \\ 2 & 1 & (s,-1) \\ 3 & 1 & (s,-1) \\ 4 & 1 & (s,-1) \\ 5 & 1 & \left(0, s^{2}\right) \\ 6 & (s, s) & 1 \\ & (-1)^{F_{R}} & (-1)^{F_{L}}\end{array}$

$s$ refers to a shift by $R / 2=l_{s} / 2 \sqrt{2}$. As explained in [2], this model has vanishing 1-loop vacuum energy (after integrating over $\operatorname{Re} \tau$ ) due to the fact that the trace of $G$ acting on level matched states in the $F$-twisted sector vanishes, 2 and the other modular inequivalent contributions vanish by zero modes due to effective supersymmetry.

2 The relevant fundamental domain for the integral is the strip instead of the normal keyhole region due to the shifts in $G$. 


\section{2-loops with supersymmetry}

In this section we review the supersymmetric cancellation at 2 loops. As explained for example in [13, 11, 12, the type II string path integral can be written as

$$
\int_{\mathcal{S} \mathcal{M}_{h}} d \mu_{0} \int[d B d C d X] e^{-S} \prod_{r=1}^{6 h-6}\left(\eta_{r}, B\right) \prod_{a=1}^{4 h-4} \delta\left(\left(\eta_{a}, B\right)\right)
$$

Here $B, C$ denote the $b, \beta$ and $c, \gamma$ ghosts, where $(b, c)$ are the spin- $(2,-1)$ conformal ghosts and $(\beta, \gamma)$ are the spin- $(3 / 2,-1 / 2)$ superconformal ghosts. $X$ denotes the matter fields and $\eta_{r}$ and $\eta_{a}$ are Beltrami differentials relating the metric and gravitino to the moduli and supermoduli. In components,

$$
\begin{aligned}
& \left(\eta_{r}, B\right)=\int \eta_{r \bar{z}}^{z} b_{z z}+\int \eta_{r \bar{z}}^{+} \beta_{z+}+\int \eta_{r z}^{\bar{z}} b_{\bar{z} \bar{z}}+\int \eta_{r z}^{-} b_{\bar{z}-} \\
& \left(\eta_{a}, B\right)=\int \eta_{a \bar{z}}^{z} b_{z z}+\int \eta_{a \bar{z}}^{+} \beta_{z+}+\int \eta_{a z}^{\bar{z}} b_{\bar{z} \bar{z}}+\int \eta_{a z}^{-} b_{\bar{z}-}
\end{aligned}
$$

As explained e.g. in [14,13], we can write the path integral measure on supermoduli space in terms of a fixed measure on moduli space

$$
d \mu_{0}=d \mu[\operatorname{sdet}(\eta, \Phi)]^{-1}[\operatorname{sdet}(\Phi, \Phi)]^{1 / 2}
$$

Here $d \mu$ is a fixed measure on the supermoduli space $\mathcal{S M}_{h}$, integrated over a fixed domain independent of the beltrami differentials. $\Phi$ contains the $3 h-3$ holomorphic and $3 h-3$ antiholomorphic 2-differentials ( $b$ ghost zero mode wavefunctions) and the $2 h-2$ holomorphic and $2 h-2$ antiholomorphic $3 / 2$-differentials ( $\beta$ ghost zero modes).

After choosing delta-function support for the worldsheet gravitinos, and integrating out the supermoduli, one obtains a correlation function of picture changing operators [11]

$$
: e^{\phi} T_{F}:=c \partial \xi+\frac{1}{2} e^{\phi} \psi^{\mu} \partial X^{\mu}-\frac{1}{4} \partial \eta e^{2 \phi} b-\frac{1}{4} \partial\left(\eta e^{2 \phi} b\right)
$$

and other ghost insertions

$$
\begin{array}{r}
\sum_{\alpha, \beta, t w i s t s} \int d \mu[\operatorname{sdet}(\eta, \Phi)]^{-1}[\operatorname{sdet}(\Phi, \Phi)]^{1 / 2}[d X][d B][d C] e^{-S}(\hat{\eta}, b)^{6 h-6} \xi\left(x_{0}\right) \\
\prod_{a=1}^{2 h-2}: e^{\phi} T_{F}\left(z_{a}\right): \prod_{a=2 h-3}^{4 h-4}: e^{\bar{\phi}} \bar{T}_{F}\left(z_{a}\right): \bar{\xi}\left(\bar{y}_{0}\right)
\end{array}
$$


The superconformal ghosts $\beta=\partial \xi e^{-\phi}, \gamma=\eta e^{\phi}$ are defined in terms of spin-0 and spin -1 fermions $\xi, \eta$ and a scalar $\phi$ [15]. The spin-0 fermion $\xi$ has a zero mode on the surface which is absorbed by the insertion of $\xi\left(x_{0}\right)$ in (3.6). There is an anomaly in the ghost number $U(1)$ current which requires insertions of operators with total ghost number $2 h-2$ to get a nonvanishing result. The correlation functions (3.6) can be evaluated using the formulas derived in e.g. [16, 17].

We will be interested in a gauge choice for which ultimately $z_{1}, z_{2} \rightarrow \Delta_{\gamma}$, where $\Delta_{\gamma}$ is a divisor corresponding to an odd spin structure $\gamma$. We need to consider the (leftmoving) spin-structure-dependent pieces of the correlation function, the poles arising from the spin-structure-independent local behavior of the picture changing correlator, and the behavior of the determinant (3.4) in this gauge. According to [11] we have the following contributions to the spin-structure-dependent pieces of the 2-loop partition function. The matter part of $T_{F}$ contributes

$$
\sum_{\delta}\langle\delta \mid \gamma\rangle \frac{\theta[\delta]^{4}(0) \theta[\delta]\left(z_{1}-z_{2}\right)}{\theta[\delta]\left(z_{1}+z_{2}-2 \Delta_{\gamma}\right)}
$$

Here $\delta \equiv(\alpha, \beta)$ encodes the spin structure of the various contributions and $\langle\delta \mid \gamma\rangle=$ $e^{4 \pi i\left(\alpha \gamma_{2}-\beta \gamma_{1}\right)}$ encodes the GSO phases [10].

Let us first, following [7], take $z_{1}+z_{2}=2 \Delta_{\gamma}$, that is place $z_{1}+z_{2}$ at a divisor corresponding to the canonical class, without setting $z_{1}=z_{2}$. The contribution (3.7) then simplifies to

$$
\sum_{\delta}\langle\delta \mid \gamma\rangle \theta[\delta]^{3}(0) \theta[\delta]\left(z_{1}-z_{2}\right)=4 \theta[\gamma]^{4}\left(z_{1}-\Delta_{\gamma}\right)
$$

where in the last step we have used a Riemann identity. The Riemann Vanishing Theorem then implies that this vanishes identically as a function of $z_{1}$. Thus in this case whatever poles arise as $z_{1} \rightarrow z_{2}$, the identical zero from the spin structure sum cancels it.

Now turning to the ghost piece of the correlation function of picture-changing operators, one obtains contributions isomorphic to (3.8) as well as

$$
\omega_{i}\left(z_{1}\right) \frac{\theta[\delta]^{5}(0) \partial_{i} \theta[\delta]\left(2 z_{2}-2 \Delta_{\gamma}\right)}{\theta^{2}[\delta]\left(z_{1}+z_{2}-2 \Delta_{\gamma}\right)}
$$

Here $\omega_{i}$ are the canonical basis of holomorphic one-forms on the Riemann surface, satisfying $\int_{a_{i}} \omega_{j}=\delta_{i j}$ and $\int_{b_{i}} \omega_{j}=\tau_{i j}$ where $\tau$ is the period matrix for the surface. Again simplifying this by first taking $z_{1}+z_{2}=2 \Delta_{\gamma}$ we obtain

$$
\sum_{\delta}\langle\delta \mid \gamma\rangle \partial_{z_{1}}\left(\theta[\delta]^{3}(0) \theta[\delta]\left(z_{1}-z_{2}\right)\right)=4 \partial_{z_{1}}\left(\theta[\gamma]^{4}\left(z_{1}-\Delta_{\gamma}\right)\right)
$$


Because the right-hand side of this expression is a derivative of 0 (by the Riemann vanishing theorem), it vanishes identically. Again any poles from the picture changing OPEs are irrelevant [7].

\section{2-loop cancellation in $F, G$ Model}

Let us consider the left-moving spin-structure-dependent pieces of the 2-loop partition function in the model of section 2. As explained in [1], all supersymmetry-breaking twist structures map by modular transformations to a canonical one in which there are twists $(1,1, F, G)$ in going around cycles $\left(a_{1}, a_{2}, b_{1}, b_{2}\right)$. The $F$ twist, which just involves an action of $(-1)^{F_{R}}$ and a symmetric shift, does not affect the $\theta$-function characteristics and phases. The effect of the $G$ twist is via its $(-1)^{F_{L}}$ action. This changes the $G$ eigenvalues by a factor of $(-1)$ in the left-moving Ramond sector relative to a model without the $(-1)^{F_{L}}$. In our twist structure there is a $G$ operator inserted on the second handle. So the effect of the $G$ twist is to change the phase of terms in the spin structure sum with $\alpha_{2}=1 / 2$, that is terms with Ramond sector states propagating around handle 2 . This means that instead of phases $\langle\delta \mid \gamma\rangle$ we should have phases $\langle\delta \mid \gamma+(0,0,0,1 / 2)\rangle$. We will set $\kappa \equiv \gamma+(0,0,0,1 / 2)$ to simplify the formulas.

Then after imposing $z_{1}+z_{2}=2 \Delta_{\gamma}$, we get for the matter correlator

$$
\begin{aligned}
\sum_{\delta}\langle\delta \mid \kappa\rangle \theta[\delta]^{3}(0) & \theta[\delta]\left(z_{1}-z_{2}\right) \theta[\gamma]\left(\sum_{i=1}^{3} w_{i}-3 \Delta_{\gamma}\right) \frac{\prod_{i<j} E\left(w_{i}, w_{j}\right)}{E\left(z_{1}, z_{2}\right)^{2}} \\
\times & \left(2 \pi p_{i}^{\mu} p_{j}^{\mu} \omega_{i}\left(z_{1}\right) \omega_{j}\left(z_{2}\right)+10 \partial_{z_{1}} \partial_{z_{2}} \log E\left(z_{1}, z_{2}\right)\right)
\end{aligned}
$$

where $E\left(z_{1}, z_{2}\right)$ is the prime form, which has the property that it goes like $z_{1}-z_{2}$ as $z_{1} \rightarrow z_{2}$. Here the $w_{i}, i=1, \ldots, 3 h-3$ are the insertion points for the $b$-ghosts. In the full amplitude [11], they are contracted with beltrami differentials encoding the gauge choice for the worldsheet metric. The spin-structure-dependent pieces here (i.e. those that depend on the characteristic $\delta$ ) sum up to

$$
4 \theta[\kappa]\left(z_{1}-\Delta_{\gamma}\right)^{4}
$$

Because of the shift in the theta characteristic after the spin structure sum, the right hand side no longer vanishes identically. However, if we choose the reference spin structure $\gamma$ such that both $\gamma$ and $\kappa \equiv \gamma+(0,0,0,1 / 2)$ are odd, then we can impose $z_{1}, z_{2} \rightarrow \Delta_{\gamma}$ and 
the right hand side of (4.2) vanishes like $\left(z_{1}-z_{2}\right)^{4}$ as $z_{1} \rightarrow z_{2}$. As $z_{1} \rightarrow z_{2}$ the determinant factor (3.4) produces another zero: plugging in the delta function $\eta_{a}$ we obtain

$$
[\operatorname{sdet}(\eta, \Phi)]^{-1} \propto \operatorname{det}\left(\eta_{a}, \Phi_{b}^{3 / 2}\right)=\operatorname{det}\left(\Phi_{b}^{3 / 2}\left(z_{a}\right)\right)
$$

Here $\Phi_{b}^{3 / 2}, b=1,2$ form a basis of holomorphic 3/2-differentials. As the $z_{a}$ approach each other, the determinant (4.3) goes to zero.

Combining the spin structure sum with the determinant factor we have a fifth order zero. As we take the limit $z_{1} \rightarrow z_{2}$ we must take into account the poles arising from the prime form $E\left(z_{1}, z_{2}\right)$ (which goes like $z_{1}-z_{2}$ as $\left.z_{1} \rightarrow z_{2}\right)$. The $2 \pi p_{i}^{\mu} p_{j}^{\mu} \omega_{i}\left(z_{1}\right) \omega_{j}\left(z_{2}\right)$ term in (4.1) has a second order pole, which is beaten by our fifth order zero. The other contributions yield

$$
\operatorname{det}\left(\eta_{a}, \Phi_{b}^{3 / 2}\right) 4 \theta[\kappa]\left(\frac{1}{2}\left(z_{1}-z_{2}\right)\right)^{4} \theta[\gamma]\left(\sum w-3 \Delta_{\gamma}\right) \prod_{i<j} E\left(w_{i}, w_{j}\right) \frac{10}{\left(z_{1}-z_{2}\right)^{4}}
$$

The poles arise from the correlator of picture changing operators. The leading singularity in this correlator is proportional to $\frac{c}{\left(z_{1}-z_{2}\right)^{4}}$ where $c$ is the total central charge (matter+ghost). Since this vanishes, there should be no such contribution after including the ghost terms in the picture changing operators. We will see this cancellation after computing the ghost correlators. In any case our fifth order zero suffices to cancel this contribution even before including the ghost piece, but after doing the spin structure sum.

The ghost correlator

$$
\begin{array}{r}
\left\langle-\frac{1}{4} c \partial \xi\left(z_{1}\right)\left(2 \partial \eta e^{2 \phi} b+\eta \partial e^{2 \phi} b+\eta e^{2 \phi} \partial b\right)\left(z_{2}\right)\right\rangle \\
+\left\langle-\frac{1}{4}\left(2 \partial \eta e^{2 \phi} b+\eta \partial e^{2 \phi} b+\eta e^{2 \phi} \partial b\right)\left(z_{1}\right) c \partial \xi\left(z_{2}\right)\right\rangle .
\end{array}
$$

gives rise to three types of contributions (see [11] eqn. (38-39) for the complete expression). One is

$$
\sum_{\delta}\langle\kappa \mid \delta\rangle \frac{\theta[\delta](0)^{5} \theta[\delta]\left(2 z_{2}-2 \Delta_{\gamma}\right) \omega^{i}\left(z_{1}\right) \partial^{i} \theta[\gamma]\left(z_{1}-z_{2}+\sum w-3 \Delta_{\gamma}\right)}{\theta[\delta]\left(z_{1}+z_{2}-2 \Delta_{\gamma}\right)^{2}} \frac{\prod_{i<j} E\left(w_{i}, w_{j}\right)}{E\left(z_{1}, z_{2}\right)^{3}}
$$

Setting $z_{1}+z_{2}=2 \Delta_{\gamma}$ and summing over $\delta$ we get for the spin-structure-dependent piece:

$$
4 \theta[\kappa]\left(\frac{1}{2}\left(z_{1}-z_{2}\right)\right)^{4} \sim\left(z_{1}-z_{2}\right)^{4}
$$


In this contribution, there is a third-order pole from the prime forms, but a fifth-order zero (4.7) from the spin structure sum times the determinant factor (4.3), so the contribution cancels as $z_{1} \rightarrow z_{2}$.

The second ghost contribution takes the form

$$
\begin{aligned}
& \sum_{\delta}\langle\kappa \mid \delta\rangle \frac{\theta[\delta](0)^{5} \theta[\delta]\left(2 z_{2}-2 \Delta_{\gamma}\right) \theta[\gamma]\left(z_{1}-z_{2}+\sum w-3 \Delta_{\gamma}\right)}{\theta[\delta]\left(z_{1}+z_{2}-2 \Delta_{\gamma}\right)^{2}} \\
& \times \frac{\prod_{i<j} E\left(w_{i}, w_{j}\right)}{E\left(z_{1}, z_{2}\right)^{3}} \partial_{z_{1}} \log \left(\frac{\prod_{j} E\left(z_{1}, w_{i}\right)}{E\left(z_{1}, z_{2}\right)^{5} \sigma\left(z_{1}\right)}\right)+\left(z_{1} \leftrightarrow z_{2}\right)
\end{aligned}
$$

where $\sigma\left(z_{1}\right)$ is defined in [1] and has no zeroes or poles. Taking $z_{1}+z_{2}=2 \Delta_{\gamma}$ and summing over $\delta$ we get

$$
4 \theta[\kappa]\left(\frac{1}{2}\left(z_{1}-z_{2}\right)\right)^{4} \frac{\prod_{i<j} E\left(w_{i}, w_{j}\right)}{E\left(z_{1}, z_{2}\right)^{3}} \partial_{z_{1}} \log \left(\frac{\prod_{j} E\left(z_{1}, w_{i}\right)}{E\left(z_{1}, z_{2}\right)^{5} \sigma\left(z_{1}\right)}\right)+\left(z_{1} \leftrightarrow z_{2}\right)
$$

This contribution has again a fifth order zero from the spin-structure sum times the determinant (as in (4.7)). There is also a fourth-order pole from the prime forms. The contribution is

$$
\begin{aligned}
& \operatorname{det}\left(\Phi_{b}^{3 / 2}\left(z_{a}\right)\right) 4 \theta[\kappa]\left(\frac{1}{2}\left(z_{1}-z_{2}\right)\right)^{4} \prod_{i<j} E\left(w_{i}, w_{j}\right) \frac{-5}{\left(z_{1}-z_{2}\right)^{4}} \theta[\gamma]\left(\sum w-3 \Delta_{\gamma}\right) \\
& +\left(z_{1} \leftrightarrow z_{2}\right)
\end{aligned}
$$

This piece cancels against the matter contribution (4.4). As mentioned above this encodes the fact that the total central charge vanishes. Again taking into account (4.3) we in fact obtain cancellation directly.

Now let us consider the remaining ghost contributions. These arise from the correlator

$$
\left\langle c \partial \xi\left(z_{1}\right)\left(\partial e^{2 \phi}\right) \eta b\left(z_{2}\right)\right\rangle+\left\langle\left(\partial e^{2 \phi}\right) \eta b\left(z_{1}\right) c \partial \xi\left(z_{2}\right)\right\rangle
$$

They take the form

$$
\begin{aligned}
& \operatorname{det}\left(\Phi_{b}^{3 / 2}\left(z_{a}\right)\right) \sum_{\delta}\langle\kappa \mid \delta\rangle \frac{\theta[\delta](0)^{5} \omega_{i}\left(z_{1}\right) \partial^{i} \theta[\delta]\left(2 z_{2}-2 \Delta_{\gamma}\right) \theta[\gamma]\left(z_{1}-z_{2}+\sum w-3 \Delta_{\gamma}\right)}{\theta[\delta]\left(z_{1}+z_{2}-2 \Delta_{\gamma}\right)^{2}} \frac{\prod_{i<j} E\left(w_{i}, w_{j}\right)}{E\left(z_{1}, z_{2}\right)^{3}} \\
& +\left(z_{1} \leftrightarrow z_{2}\right)
\end{aligned}
$$

In the first term of this contribution (4.11), setting $z_{1}+z_{2}=2 \Delta_{\gamma}$ and summing on $\delta$ gives

$$
\omega_{i}\left(\Delta_{\gamma}\right) \partial^{i} \theta[\kappa]\left(z_{1}-z_{2}\right)^{4} \frac{1}{\left(z_{1}-z_{2}\right)^{3}}
$$


plus other terms which have lower-order poles. The spin structure sum only gives a third order zero here due to the derivative acting on the theta functions. The $\operatorname{det}\left(\Phi_{b}^{3 / 2}\left(z_{a}\right)\right)$ factor gives an additional zero which kills this contribution as $z_{1} \rightarrow z_{2}$.

Thus we see that in the $(F, G)$ model we can pick a gauge for the worldsheet metric as well as gravitino, for which all the contributions cancel, after summing over all spin structures and using appropriate Riemann identities. Note that the cancellations explained above occur via the spin structure sum of each term in the correlator of picture-changing operators separately, so that the shifts twisting some of the bosons do not affect the argument. The above cancellation alone is not enough to guarantee that the two-loop amplitude cancels; one needs the cancellation of the boundary contributions that potentially arise due to the ambiguity in the choice of gauge slice for the gravitini [18]. In the $(F, G)$ model these appear to cancel similarly to the analyis in the original models [1]. Note that although the bulk cancellations we exhibit here would hold also for example for the type of model studied in [19] (generated by a translation combined with $(-1)^{F_{L}+F_{R}}$ ), those models have a nonzero one-loop vacuum energy and non-zero one-loop tadpoles (as well as tachyons, for some range of the moduli VEVs). Therefore, the boundary contributions in the latter case would destroy the cancellation. Nonetheless it is somewhat surprising that the bulk contribution can be made to cancel by choosing an appropriate gauge in that model.

\section{Taylor Expansion in Original Models}

In this section we will present the analysis of the coincident gauge for the original models of [1] (see that paper for the definition of the model). As in [1], we only need to analyze the twist structure $(1,1, f, g)$. The $f$ twist affects the characteristics of some of the $\theta$ functions (arising from twisted fields) by shifting them by $(0,0,1 / 2,0)$ - we shall denote this as a shift by $\frac{1}{2} L . \kappa$ is, as in $\S 4, \gamma+(0,0,0,1 / 2)$, and we choose $\gamma$ such that both $\gamma$ and $\kappa$ are odd.

The correlation function of the matter part of the picture changing operators breaks into two contributions. The terms involving $\left\langle\psi^{i} \partial X^{i}\left(z_{1}\right) \psi^{i} \partial X^{i}\left(z_{2}\right)\right\rangle$ with $i=5, \cdots 10$ give

$$
\begin{array}{r}
\sum_{\delta}\langle\kappa \mid \delta\rangle \frac{\theta[\delta](0)^{2} \theta\left[\delta+\frac{1}{2} L\right](0)^{2} \theta[\delta]\left(z_{1}-z_{2}\right)}{\theta[\delta]\left(z_{1}+z_{2}-2 \Delta_{\gamma}\right)} \\
\times\left(p_{i}^{\mu} \omega^{i}\left(z_{1}\right) p_{j}^{\mu} \omega^{j}\left(z_{2}\right) \frac{1}{E\left(z_{1}, z_{2}\right)^{2}}+\frac{6}{E\left(z_{1}, z_{2}\right)^{2}} \partial_{z_{1}} \partial_{z_{2}} \log E\left(z_{1}, z_{2}\right)\right) \\
\times \operatorname{det}\left(\Phi_{a}^{3 / 2}\left(z_{b}\right)\right)
\end{array}
$$


Upon setting $z_{1}+z_{2}=2 \Delta_{\gamma}$ we can cancel the denominator against one factor in the numerator to get

$$
\sum_{\delta}\langle\kappa \mid \delta\rangle \theta[\delta](0) \theta[\delta]\left(z_{1}-z_{2}\right) \theta\left[\delta+\frac{1}{2} L\right](0)^{2}=4 \theta[\kappa]\left(\frac{1}{2}\left(z_{1}-z_{2}\right)\right) \theta\left[\kappa+\frac{1}{2} L\right]\left(\frac{1}{2}\left(z_{1}-z_{2}\right)\right)
$$

for the spin-dependent piece of this correlator. Because $\kappa$ is an odd spin structure, this vanishes like $\left(z_{1}-z_{2}\right)^{2}$. The factor of $\operatorname{det}\left(\Phi_{a}^{3 / 2}\left(z_{b}\right)\right)$ in (5.1) also vanishes like $\left(z_{1}-z_{2}\right)$ as $z_{1} \rightarrow z_{2}$, so all in all (5.1) has a $\left(z_{1}-z_{2}\right)^{3}$ multiplying the prime forms. However, since the prime-forms are yielding poles as $z_{1} \rightarrow z_{2}$, it remains to check that there are no finite pieces in (5.1).

The terms proportional to $\frac{1}{E\left(z_{1}, z_{2}\right)^{2}}$ times the loop momenta clearly vanish in the limit, since there is only a second order pole from the prime forms which cannot cancel the third order zero we found from the spin structure sum and the superdeterminant. This leaves the term which goes like $\frac{1}{E\left(z_{1}, z_{2}\right)^{2}} \partial_{z_{1}} \partial_{z_{2}} \log E\left(z_{1}, z_{2}\right)$. Using the fact that $E\left(z_{1}, z_{2}\right)$ has a Taylor expansion of the form

$$
E\left(z_{1}, z_{2}\right) \sim \sum_{n=0}^{\infty} c_{n}\left(z_{1}-z_{2}\right)^{2 n+1}
$$

as $z_{1} \rightarrow z_{2}$, one sees that this combination of prime forms has an expansion

$$
\frac{1}{E\left(z_{1}, z_{2}\right)^{2}} \partial_{z_{1}} \partial_{z_{2}} \log E\left(z_{1}, z_{2}\right) \sim \sum_{n=-2}^{\infty} d_{n}\left(z_{1}-z_{2}\right)^{2 n}
$$

On the other hand, the determinant factor is an odd function of $z_{1}-z_{2}$ with an expansion of the form

$$
\operatorname{det}\left(\Phi_{a}^{3 / 2}\left(z_{b}\right)\right) \sim \sum_{m=0}^{\infty} e_{m}\left(z_{1}-z_{2}\right)^{2 m+1}
$$

while the sum over spin structures (5.2) is an even function with a second order zero at $z_{1}=z_{2}$. From these facts, it is easy to see that the full expression (5.1) has an expansion of the form

$$
\sum_{j=0}^{\infty} f_{j}\left(z_{1}-z_{2}\right)^{2 j-1}
$$

as $z_{1} \rightarrow z_{2}$.

Examining (5.6), we see that

- There are no finite contributions as $z_{1} \rightarrow z_{2}$. 
- There is a (gauge artifact) pole as $z_{1} \rightarrow z_{2}$; in fact this pole receives contributions from the various matter and ghost correlators proportional to the matter/ghost central charges, and hence cancels once all of the terms are taken into account $\left(\right.$ since $\left.c_{\text {tot }}=c_{\text {matter }}+c_{\text {ghost }}=0\right)$. We will see this explicitly once we compute the remaining matter and ghost contributions.

The second type of matter correlator arises from contracting the $\psi^{i} \partial X^{i}\left(z_{1}\right) \psi^{i} \partial X^{i}\left(z_{2}\right)$ with $i=1, \cdots 4$. This leads to a contribution

$$
\begin{array}{r}
\sum_{\delta}\langle\kappa \mid \delta\rangle \frac{\theta[\delta](0)^{3} \theta\left[\delta+\frac{1}{2} L\right](0) \theta\left[\delta+\frac{1}{2} L\right]\left(z_{1}-z_{2}\right)}{\theta[\delta]\left(z_{1}+z_{2}-2 \Delta_{\gamma}\right)} \\
\times\left(p_{i}^{\mu} \omega^{i}\left(z_{1}\right) p_{j}^{\mu} \omega^{j}\left(z_{2}\right) \frac{1}{E\left(z_{1}, z_{2}\right)^{2}}+\frac{4}{E\left(z_{1}, z_{2}\right)^{2}} \partial_{z_{1}} \partial_{z_{2}} \log E\left(z_{1}, z_{2}\right)\right) \\
\times \operatorname{det}\left(\Phi_{a}^{3 / 2}\left(z_{b}\right)\right)
\end{array}
$$

Choosing $z_{1}+z_{2}=2 \Delta_{\gamma}$, the spin sum in (5.7) simplifies to

$$
\sum_{\delta}\langle\kappa \mid \delta\rangle \theta[\delta](0)^{2} \theta\left[\delta+\frac{1}{2} L\right](0) \theta\left[\delta+\frac{1}{2} L\right]\left(z_{1}-z_{2}\right)
$$

which, after applying a Riemann identity, becomes

$$
4 \theta[\kappa]\left(\frac{1}{2}\left(z_{1}-z_{2}\right)\right)^{2} \theta\left[\kappa+\frac{1}{2} L\right]\left(\frac{1}{2}\left(z_{1}-z_{2}\right)\right)^{2}
$$

So in fact after summing over spin structures this looks the same as the spin sum of the first type of matter contribution (5.2). Again, it vanishes like $\left(z_{1}-z_{2}\right)^{2}$ as $z_{1} \rightarrow z_{2}$.

Now, the argument for the cancellation proceeds as it did for the first type of matter contribution. The terms involving only the $\frac{1}{E\left(z_{1}, z_{2}\right)^{2}}$ multiplying loop momenta only have a second order pole, which cannot cancel the third order zero coming from the determinant times the spin structure sum (5.9). The terms involving higher inverse powers of the prime forms lead to a simple pole (which cancels after summing over matter and ghosts, as it is proportional to the total central charge) and no finite contributions.

Next, let us consider the terms in the correlator of picture changing operators coming from the ghost part of the worldsheet supercurrent (4.5). There are three types of terms that arise [11]. We are in the twist structure $(1,1, f, g)$. As in the matter sector, the $f$ twist affects the characteristics of the $\theta$-functions arising in the worldsheet correlation functions 
and determinants. We will denote the shift in the characteristic, which is $(0,0,1 / 2,0)$, as $\frac{1}{2} L$. The first type of contribution is

$$
\begin{array}{r}
\sum_{\delta}\langle\kappa \mid \delta\rangle \frac{\theta[\delta](0)^{3} \theta\left[\delta+\frac{1}{2} L\right](0)^{2} \theta[\delta]\left(2 z_{2}-2 \Delta_{\gamma}\right) \theta\left(z_{1}-z_{2}+\sum w-3 \Delta\right)}{\theta[\delta]\left(z_{1}+z_{2}-2 \Delta_{\gamma}\right)^{2} E\left(z_{1}, z_{2}\right)^{3}} \\
\times \operatorname{det}\left(\Phi_{a}\left(z_{b}\right)\right) \frac{\prod E\left(z_{1}, w\right)}{\prod E\left(z_{2}, w\right)} \partial_{z_{1}} \log \left(\frac{\prod E\left(z_{1}, w\right)}{E\left(z_{1}, z_{2}\right)^{5} \sigma\left(z_{1}\right)}\right) \\
+\left(z_{1} \leftrightarrow z_{2}\right)
\end{array}
$$

The second is

$$
\begin{array}{r}
\sum_{\delta}\langle\kappa \mid \delta\rangle \frac{\theta[\delta](0)^{3} \theta\left[\delta+\frac{1}{2} L\right](0)^{2} \omega_{i}\left(z_{1}\right) \partial^{i} \theta[\delta]\left(2 z_{2}-2 \Delta_{\gamma}\right) \theta\left(z_{1}-z_{2}+\sum w-3 \Delta\right)}{\theta[\delta]\left(z_{1}+z_{2}-2 \Delta_{\gamma}\right)^{2} E\left(z_{1}, z_{2}\right)^{3}} \\
\times \operatorname{det}\left(\Phi_{a}\left(z_{b}\right)\right) \frac{\prod E\left(z_{1}, w\right)}{\prod E\left(z_{2}, w\right)} \\
+\left(z_{1} \leftrightarrow z_{2}\right)
\end{array}
$$

The third is

$$
\begin{array}{r}
\sum_{\delta}\langle\kappa \mid \delta\rangle \frac{\theta[\delta](0)^{3} \theta\left[\delta+\frac{1}{2} L\right](0)^{2} \theta[\delta]\left(2 z_{2}-2 \Delta_{\gamma}\right) \omega_{i}\left(z_{1}\right) \partial^{i} \theta\left(z_{1}-z_{2}+\sum w-3 \Delta\right)}{\theta[\delta]\left(z_{1}+z_{2}-2 \Delta_{\gamma}\right)^{2} E\left(z_{1}, z_{2}\right)^{3}} \\
\times \operatorname{det} \Phi_{a}\left(z_{b}\right) \frac{\prod E\left(z_{1}, w\right)}{\prod E\left(z_{2}, w\right)} \\
+\left(z_{1} \leftrightarrow z_{2}\right)
\end{array}
$$

Setting $z_{1}+z_{2}=2 \Delta_{\gamma}$ and doing the spin structure sum we find for the spin-structuredependent pieces of contributions (5.10) and (5.12):

$$
\begin{array}{r}
\sum_{\delta}\langle\kappa \mid \delta\rangle \theta[\delta](0) \theta\left[\delta+\frac{1}{2} L\right](0)^{2} \theta[\delta]\left(2 z_{2}-2 \Delta_{\gamma}\right) \\
=\theta[\kappa]\left(z_{2}-\Delta_{\gamma}\right)^{2} \theta\left[\kappa+\frac{1}{2} L\right]\left(z_{2}-\Delta_{\gamma}\right)^{2} \\
\sim\left(z_{1}-z_{2}\right)^{2}+c_{4}\left(z_{1}-z_{2}\right)^{4}+\ldots
\end{array}
$$

for some constant $c_{4}$ where in the last line we expanded the result in a Taylor expansion around $z_{1}=z_{2}$. For contribution (5.11) we get

$$
\begin{array}{r}
\sum_{\delta}\langle\kappa \mid \delta\rangle \theta[\delta](0) \theta\left[\delta+\frac{1}{2} L\right](0)^{2} \omega_{i}\left(z_{1}\right) \partial^{i} \theta[\delta]\left(2 z_{2}-2 \Delta_{\gamma}\right) \\
=\partial_{z_{1}}\left(\theta[\kappa]\left(\frac{1}{2}\left(z_{1}-z_{2}\right)\right)^{2} \theta\left[\kappa+\frac{1}{2} L\right]\left(\frac{1}{2}\left(z_{1}-z_{2}\right)\right)^{2}\right) \\
\sim\left(z_{1}-z_{2}\right)+b_{3}\left(z_{1}-z_{2}\right)^{3}+\ldots
\end{array}
$$


As for the matter contributions, although the spin structure sums give vanishing contributions, they multiply singularities arising from the prime forms $E\left(z_{1}, z_{2}\right)$ and we must analyze the potential finite terms in the Taylor expansion. Let us consider first (5.10). There are two types of contributions here. After doing the spin structure sum as above the first takes the form

$$
-5 \frac{\partial_{1} E\left(z_{1}, z_{2}\right)}{E\left(z_{1}, z_{2}\right)^{4}}\left[z_{12}^{2}+c_{4} z_{12}^{4}+\ldots\right]\left[z_{12}+e_{3} z_{12}^{3}+\ldots\right] \frac{\prod E\left(z_{1}, w\right)}{\prod E\left(z_{2}, w\right)}+\left(z_{1} \leftrightarrow z_{2}\right)
$$

where we denote $z_{1}-z_{2}$ by $z_{12}$. Here the second factor comes from the spin structure sum, the third from the Taylor expansion of the determinant about $z_{1}=z_{2}$ (where $e_{3}$ is some constant). We should emphasize what is meant here by $\left(z_{1} \leftrightarrow z_{2}\right)$. We are computing a correlation function of picture changing operators. The ghost piece of this correlator has the form (4.5). So for example the second term in (4.5) corresponds to the term denoted $z_{1} \leftrightarrow z_{2}$ in (5.15). So in particular the second term involves interchanging the operators in the ghost correlator, without changing $z_{1}$ to $z_{2}$ in the determinant factor. The first and fourth factors involving the prime forms encode the physical poles and zeroes of the correlator. The leading singularity from the prime forms here comes from the $1 / z_{12}^{4}$ term in the expansion of the prime form factors. Therefore only the leading term in the Taylor expansion of the spin structure sum and determinant factors potentially survive (so we can ignore the terms proportional to $c_{4}$ or $e_{3}$, which give fifth-order zeroes). Similarly expanding the prime forms $E\left(z_{1}, z_{2}\right)$ gives a subleading term with only a $1 / z_{12}^{2}$ pole, which is cancelled by the third order zero coming from the leading piece of the spin structure sum times determinant.

Putting the factors together, we see that the leading piece is a simple pole in $z_{12}$. The first three factors in (5.15) are the same in the term with $z_{1} \leftrightarrow z_{2}$. When we include the term with $z_{1} \leftrightarrow z_{2}$, they multiply the prime form factor $\frac{\prod E\left(z_{1}, w\right)}{\prod E\left(z_{2}, w\right)}+\frac{\prod E\left(z_{2}, w\right)}{\prod E\left(z_{1}, w\right)}$. This is even under $z_{1} \leftrightarrow z_{2}$. In our Taylor expansion it therefore becomes of the form $\mathcal{O}(1)+f_{2} z_{12}^{2}+\ldots$, and only the first term contributes. Therefore in Taylor expanding the contribution (5.15), we get a pole piece plus higher order terms which vanish in the limit $z_{1} \rightarrow z_{2}$. In particular, no finite pieces survive. What is the interpretation of the pole piece? It is proportional to the ghost central charge, and precisely cancels the pole piece coming from the matter contribution.

The second type of contribution in (5.10) takes the form

$$
\frac{1}{E\left(z_{1}, z_{2}\right)^{3}}\left[z_{12}^{2}+\ldots\right]\left[z_{12}+\ldots\right] \frac{\prod E\left(z_{1}, w\right)}{\prod E\left(z_{2}, w\right)} \partial_{1} \log \left(\frac{\prod E\left(z_{1}, w\right)}{\sigma\left(z_{1}\right)}\right)
$$


where the ... denotes terms which vanish automatically as $z_{1} \rightarrow z_{2}$. The leading pole from the prime forms here is cubic. Before including the $z_{1} \leftrightarrow z_{2}$ term there is a finite piece obtained by multiplying this times the third order zero obtained from the spin structure sum and determinant factors. The spin structure sum is even under the interchange of $z_{1}$ and $z_{2}$ in this case, and as discussed above the determinant factor is the same in both terms. The factor $\frac{1}{E\left(z_{1}, z_{2}\right)^{3}}$ does change sign between the two terms, however. So when we add the $\left(z_{1} \leftrightarrow z_{2}\right)$ term the contribution cancels.

Let us now consider the contribution (5.11). This gives a contribution of the form

$$
\frac{1}{E\left(z_{1}, z_{2}\right)^{3}}\left[z_{12}+\ldots\right]\left[z_{12}+\ldots\right]\left(\frac{\prod E\left(z_{1}, w\right)}{\prod E\left(z_{2}, w\right)}+\frac{\prod E\left(z_{2}, w\right)}{\prod E\left(z_{1}, w\right)}\right)
$$

Here similarly to the above analysis we took into account the relative sign of the two contributions in (4.5) and included the $z_{1} \leftrightarrow z_{2}$ contribution. The last factor here is even under interchange of $z_{1}$ and $z_{2}$, so its Taylor expansion is of the form $1+h_{2} z_{12}^{2}+\ldots$ for some constant $h_{2}$. The leading contribution here is a simple pole, and there is no finite contribution.

Unlike the previous simple poles we have encountered, the pole encountered here does not cancel with the other matter and ghost contributions (it is not one of the pieces which would have contributed to the $\frac{c}{z^{4}}$ pole in the OPE of picture changing operators before accounting for spin structure sums and determinant factors). However, on general grounds we expect such gauge artifact poles to constitute total derivatives on moduli space. Otherwise the invariance of the path integral on gauge slice would be lost. In this case, we can argue for that conclusion as follows. The pole we are discussing receives a $\frac{1}{\left(z_{1}-z_{2}\right)^{3}}$ contribution from the prime forms which is softened to $\frac{1}{\left(z_{1}-z_{2}\right)^{2}}$ by the theta function zero (and then to a simple pole by the determinant factor). In the OPE of picture changing operators, the $\frac{1}{\left(z_{1}-z_{2}\right)^{2}}$ divergence is multiplied by the stress-energy tensor, which gives a derivative with respect to the metric and therefore the moduli. The term we are finding is part of this total derivative. In the gauge we have chosen, it is the only non-vanishing piece (the other pieces vanish even before integration over the moduli space). However, since there cannot be gauge artifact poles, we expect it to integrate to zero (which one can argue for using the boundary analysis in [1]).

Finally let us consider the last ghost contribution (5.12). This contribution takes the form

$$
\begin{aligned}
\frac{1}{E\left(z_{1}, z_{2}\right)^{3}}\left[z_{12}^{2}+\ldots\right]\left[z_{12}+\ldots\right] & \frac{\prod E\left(z_{1}, w\right)}{\prod E\left(z_{2}, w\right)} \\
& +\left(z_{1} \leftrightarrow z_{2}\right)
\end{aligned}
$$


In this contribution before including the $z_{1} \leftrightarrow z_{2}$ contribution there is a potential finite term from the third order pole multiplying a third order zero in $z_{12}$. Here again, in the limit $z_{1} \rightarrow z_{2}$ every factor except the first is the same in the two terms. The first factor $\frac{1}{E\left(z_{1}, z_{2}\right)^{3}}$ has the opposite sign in the two terms. Thus again after including the $z_{1} \leftrightarrow z_{2}$ term the contribution cancels.

\section{Discussion}

In the original models of the type described in [1], as well as in the new model described in [2], we see here that the two loop contribution cancels in the gauge with coincident picture changing operators after properly taking into account the singularities.

This analysis, combined with the analysis of boundary contributions presented in [1], yields a vanishing two loop cosmological constant in these models. Note that we computed this contribution in a particular twist structure, in the gauge where $z_{1}$ and $z_{2}$ approach a particular branch point $\Delta_{\gamma}$. Performing a modular transformation moves us to a different twist structure-as explained in [1] one can recover all twist structures in this way-but also permutes the branch points and in general will change the gauge choice. 3 However at a given twist structure, one choice of insertion points for picture changing operators is related to another by total derivative terms which cancel in our model [1]. Therefore in a fixed gauge we are in fact not finding cancellation pointwise on the moduli space, but instead only after integration (using the formal argument of [11] to reduce the integrals to boundary contributions). A modular invariant integrand is obtained on the other hand if we let the gauge choice get modular tranformed along with the twist structure. Needless to say it would be interesting to compute the integral directly in some fixed gauge to check the cancellation independently.

\section{Acknowledgements}

We would like to thank G. Moore for pointing out the deficiency in the 2-loop computation in [1], and for extremely helpful discussions. We are also grateful to E. d'Hoker, J. Harvey, Z. Kakushadze, J. Kumar, S. Shenker, H. Verlinde and E. Witten for helpful discussions. This work was initiated at the Amsterdam Summer Workshop on String Theory and continued while E.S. was enjoying the hospitality of the Aspen Center for Physics. S.K. is supported by NSF grant PHY-94-07194, by DOE contract DE-AC03-76SF0098, by an A.P. Sloan Foundation Fellowship and by a DOE OJI Award. E.S. is supported by DOE contract DE-AC03-76SF00515.

3 We thank Z. Kakushadze for discussions on this point. 


\section{References}

[1] S. Kachru, J. Kumar and E. Silverstein, "Vacuum Energy Cancellation in a Nonsupersymmetric String," hep-th/9807076.

[2] S. Kachru and E. Silverstein, "Self-Dual Nonsupersymmetric Type II String Compactifications," hep-th/9808056.

[3] G. Shiu and S.H. Tye, "Bose-Fermi Degeneracy and Duality in Non-Supersymmetric Strings," hep-th/9808095.

[4] S. Kachru and E. Silverstein, "4d Conformal Field Theories and Strings on Orbifolds," hep-th/9802183.

[5] J. Maldacena, "The Large N Limit of Superconformal Field Theories and Supergravity," hep-th/9711200;

S. Gubser, I. Klebanov and A. Polyakov, "Gauge Theory Correlators from Noncritical String Theory," Phys. Lett. B428 (1998) 105, hep-th/9802109;

E. Witten, "Holography and Anti de Sitter Space," hep-th/9802150.

[6] J. Harvey, "String Duality and Nonsupersymmetric Strings," hep-th/9807213.

[7] O. Lechtenfeld and A. Parkes, "On Covariant Multi-loop Superstring Amplitudes," Nucl. Phys. B332 (1990) 39.

[8] A. Morozov and A. Perelomov, "Statistical Sums in Superstring Theory: Genus 2," Phys. Lett. B199 (1987) 209.

[9] A. Morozov, "Two-Loop Statsum of Superstrings," Nucl. Phys. B303 (1988) 343.

[10] O. Lechtenfeld, "Factorization and Modular Invariance of Multiloop Superstring Amplitudes in the Unitary Gauge," Nucl. Phys. B338 (1990) 403.

[11] E. Verlinde and H. Verlinde, "Mutliloop Calculations in Covariant Superstring Theory," Phys. Lett. B192 (1987) 95.

[12] J. Atick, G. Moore, and A. Sen, "Some Global Issues in String Perturbation Theory," Nucl. Phys. B308 (1988) 1.

[13] E. d'Hoker and D. Phong, "The Geometry of String Perturbation Theory," Rev. Mod. Phys. 60 (1988) 917.

[14] E. Martinec, "Conformal Field Theory on a (Super)Riemann Surface," Nucl. Phys. B281 (1987) 157.

[15] D. Friedan, E. Martinec and S. Shenker, "Conformal Invariance, Supersymmetry and String Theory," Nucl. Phys. B271 (1986) 93.

[16] E. Verlinde and H. Verlinde, "Chiral Bosonization, Determinants and the String Partition Function," Nucl. Phys. B288 (1987) 357.

[17] T. Eguchi and H. Ooguri, "Chiral Bosonization on a Riemann Surface," Phys. Lett. 187B (1987) 127.

[18] J. Atick, G. Moore and A. Sen, "Catoptric Tadpoles," Nucl. Phys. B307 (1988) 221.

[19] R. Rohm, "Spontaneous Supersymmetry Breaking in Supersymmetric String Theories," Nucl. Phys. B237 (1984) 553. 